\title{
The role of MAPK signaling pathway in the Her-2-positive meningiomas
}

\author{
ZHAOYIN WANG $^{1 *}$, WEIJIA WANG ${ }^{1 *}$, SHAN XU $^{1 *}$, SHANSHAN WANG ${ }^{1}$, \\ YI TU $^{1}$, YIFENG XIONG ${ }^{1}$, JINHONG MEI $^{1}$ and CHUNLIANG WANG ${ }^{2}$ \\ Departments of ${ }^{1}$ Pathology and ${ }^{2}$ Neurosurgery, The First Affiliated Hospital \\ of Nanchang University, Nanchang, Jiangxi 330006, P.R. China
}

Received February 13, 2016; Accepted March 18, 2016

DOI: $10.3892 /$ or.2016.4849

\begin{abstract}
Meningiomas are common types of adult nerve system tumors. Although most cases are considered benign, due to its high rate of recurrence and easy malignant progression to anaplastic meningioma they present a puzzle for the current treatment. The HER-2 oncogene has important value for meningioma cells development and progression. So far, little is known about the effect on the exact underlying signal pathway and molecular mechanisms of HER-2-positive meningioma cells. The goal of the present study was to determine the effects of HER-2 gene and possible involvement of MAPK signal pathway in human malignant meningioma. We applied q-PCR analysis, immunofluorescence (IF) staining, western blot analysis, animal model, MAPK inhibition, MTT assay and cell invasion analysis for the investigation. The results demonstrated that the downregulation of the expression of HER-2 significantly inhibited cell motility and proliferation of human meningioma cells in vivo. Accordingly, in the HER-2-overexpression meningioma cells with the inhibition of ERK1/2, ERK5, JNK, in the cells with the ERK1/2, ERK5 inhibition, protein expression was markedly suppressed as well as the cell proliferation resistance. No difference was observed in the HER-2-overexpression meningioma cells with the inhibition of JNK. These findings suggest that HER-2 gene can affect the proliferation ability of human meningioma cells in vivo and MAPK signal pathway may contribute to
\end{abstract}

Correspondence to: Professor Chunliang Wang, Department of Neurosurgery, The First Affiliated Hospital of Nanchang University, 17 Yongwai Zheng Road, Nanchang, Jiangxi 330006, P.R. China E-mail: wang330006@126.com

Professor Jinhong Mei, Department of Pathology, The First Affiliated Hospital of Nanchang University, 17 Yongwai Zheng Road, Nanchang, Jiangxi 330006, P.R. China

E-mail: mjhdoctor@126.com

*Contribute equally

Key words: human malignant meningioma, HER-2, proliferation, invasion, MAPK the carcinogenesis and development of human meningiomas combinating with HER-2.

\section{Introduction}

Meningioma is a common type tumor of the central nervous system, divided into three groups: grade I (benign meningioma), II (Atypical meningioma), III (anaplastic/malignant meningioma) (1). While grade I meningioma with its benign clinical behavior with the possibility of surgical and radiation cure, WHO grade II and III are correlated with aggressive behavior with higher risk of recurrence, metastasis and shorter survival times (2). Moreover, according to the studies, lung, bone, liver, lymph node, and kidney sites relapse frequently and occasionally undergo malignant transformation or metastasis (3). Surgery is the mainstay of treatment for patients with an atypical or malignant meningioma, these lesions are usually treated with adjuvant external beam radiotherapy (RT). However, the effect of the postoperative RT for atypical meningiomas remains controversial, bringing us challenges for the current treatment strategies of combined surgery and radiotherapy (4). Therefore, understanding the molecular mechanisms of invasion or metastasis will pave the way to the discovery of new therapeutic agents for decreasing recurrence rate and increasing prognostic accuracy in human meningioma cells.

HER2 is affiliated with the erythoblastosis oncogene B (ErbB) receptor tyrosine kinase (RTK) family, also known as ErbB2, c-erbB2 or HER2/neu, coding a $185 \mathrm{kDa}$ transmembrane tyrosine kinase receptor protein. HER2 has a key role to form a heterodimer with other HER members, mediating relevant activation of signal transduction pathway that control cell growth, differentiation, motility in normal cells as well as cell proliferation, survival, invasion, angiogenesis in tumor cells. The two main signaling pathways: phosphoinositide-3-kinase (PI3K)/AKT and rat sarcoma/mitogen-activated protein kinase (RAS/MAPK) pathways mediated by the dimerisation and transphosphorylation of RTKs $(5-7,14)$. Molecular mechanisms of HER2-overexpression have been elucidated both in breast cancers and non-breast cancers (8). Nevertheless, the role of HER2 associated with meningiomas have not been demonstrated completely (9-12). In our previous study, we provided evidence that expression of HER 2 contributed to the 
cell proliferation and invasion and inhibiting apoptosis in the human malignant IOMM-Lee cells. Upregulation of HER2 impacted the protein level of PI3K and AKT, thereby illustrating that the PI3K signal pathway play a important role in mediating invasion in the IOMM-Lee cells (13). Therefore, it is speculated that HER-2-RAS-MAPK also plays a role on the pathogenesis of malignant meningioma.

In the present study, the possible role of RAS-MAPK signal pathway in cell cycle progression and tumor invasion of the IOMM-Lee human malignant meningioma cell line was investigated. The results may open a way to provide new ideas for further investigation of the invasion and proliferation mechanism of meningiomas and to find some promising molecular targeted therapies.

\section{Materials and methods}

Cell lines and cell culture. The IOMM-Lee cell lines were incubated at a humidified $37^{\circ} \mathrm{C}$ incubator containing $5 \% \mathrm{CO}_{2}$ and grown in Dulbecco's modified Eagle's medium (DMEM; GE Healthcare, Logan, UT, USA), supplemented with $10 \%$ fetal bovine serum (FBS; Gibco-BRL, Carlsbad, CA, USA), $100 \mathrm{U} / \mathrm{ml}$ streptomycin and $100 \mathrm{U} / \mathrm{ml}$ penicillin (Beijing Solarbio Science \& Technology Co., Ltd., Beijing, China), The IOMM-Lee human meningioma cell line was kindly provided by Dr ? Jensen and Dr ? Gillespie of the University of Utah (Salt Lake City, UT, USA).

Plasmids and transfection. The acquisition of fragments of the short hairpin (sh) HER-2 (HER-2-sh) and HER-2overexpression sequence were attributed to NCBI references from HanBio (Shanghai, China) and the NM_004448 GenBank HER2 NCBI reference sequence, respectively, thereby the HER-2-sh and HER-2-overexpression lentiviral vectors were constructed. Nonsense sequence lentiviral vectors (NC-sh and NC-overexpression) were used as negative controls. The HER-2-sh lentiviral vectors were purchased from HanBio and the HER-2-overxepression lentiviral vectors were purchased from GeneChem (Shanghai, China), respectively. The IOMM-Lee cells were seeded into a 6-well plate and cells at 30-50\% confluence were infected with NC-sh, HER-2-sh, NC-overexpression, HER-2-overexpression, at a multiplicity of infection of 15 with polybrene ( $5 \mu \mathrm{g} / \mathrm{ml}$; GeneChem), then washed with fresh medium $8 \mathrm{~h}$ later. To obtain stable cell lines, selected with $2 \mu \mathrm{g} / \mathrm{ml}$ puromycin (Sigma-Aldrich, St. Louis, MO, USA) for 2 weeks. Stable transformants were examined with fluorescence microscopy, real-time quantitative polymerase chain reaction (q-PCR) and western blot analysis.

q-PCR analysis. Total RNAs were extracted from the transfected IOMM-Lee cells using TRIzol reagent (Invitrogen Life Technologies, Carlsbad, CA, USA). The RNA quality and concentration were analyzed by measuring the absorbance at 260 and $280 \mathrm{~nm}$ with the spectrometer (759S; Shanghai Lengguang Technology Co., Ltd., Shanghai, China), and the A260/A280 ratios were between 1.8 and 2.0. For singlestranded cDNA synthesis, the RT reaction was performed using a RevertAid First Strand cDNA synthesis kit (Transgen, Beijing, China). q-PCR was then performed with $1 \mu \mathrm{g}$ RNA and $1 \mu \mathrm{l}$ of the following primers from Invitrogen Life Technologies: HER-2: forward, 5'-CGGACGCCTGATGGGTTAAT-3'
(120 bp) and reverse, 5'-ACAGCAAAGGTTCTACCCCG-3'; and GAPDH: forward, 5'-CAGGGCTGCTTTTAACTCT GGT-3' (203 bp) and reverse, 5'-GATTTTGGAGGGATCTC GCT-3'. The q-PCR procedure conducted in the ABI PRISM 7500 System (Applied Biosystems, Waltham, MA, USA) was as follows: denaturing at $95^{\circ} \mathrm{C}$ for $2 \mathrm{~min}$ and 40 cycles of annealing at $95^{\circ} \mathrm{C}$ for $15 \mathrm{sec}$ and extension at $58^{\circ} \mathrm{C}$ for $30 \mathrm{sec}$.

Immunofluorescence (IF) staining. Cells were seeded on an 8-well Lab-Tek Chambered Coverglass the day prior to experiments. The slides were washed with phosphate-buffered saline (PBS) and cells were fixed with $4 \%$ paraformaldehyde, followed by permeabilization with methanol. After washing with PBS, the slides were blocked with $2 \%$ bovine serum albumin (BSA), followed by incubation with primary and secondary antibodies in 5\% BSA. Subsequently, the slides were mounted in the mounting solution (Invitrogen) containing DAPI for counterstaining cell nucleus. The cell images were observed and photographed with an immunofluorescence microscopy.

Western blot analysis. When the stable cells grew in the exponential growth phase, they were seeded into 6-well plates and allowed to grow until $80-90 \%$ confluence, following which they were lysed in lysis buffer (Beyotime Institute of Biotechnology, Beijing, China) on ice. The cells were harvested, washed twice with 1X phosphate-buffered saline (PBS) and lysed in $100 \mu \mathrm{l}$ radioimmunoprecipitation assay lysis buffer [Vazyme Biotech (Nanjing) Co., Ltd., Nanjing, China]. Protein concentrations were determined using a bicinchoninic acid kit [Vazyme Biotech (Nanjing) Co., Ltd.]. The proteins were separated using 10\% SDS-PAGE and were then blotted onto nitrocellulose membranes by wet electroblotting at a constant current $200 \mathrm{~mA}$ for $2 \mathrm{~h}$.

The membranes were blocked with $5 \%$ non-fat milk powder at room temperature for $1 \mathrm{~h}$ and incubated overnight with the following primary antibodies: monoclonal mouse HER-2 (3B5; 1:500; Abcam, Cambridge, MA, USA), polyclonal rabbit ERK5 (D315V; 1:1,000; Cell Signaling Technology, Inc., Danvers, MA, USA), polyclonal rabbit phosphorylated (p)-ERK5 (Thr218/Tyr220; 1:1,000; Cell Signaling Technology, Inc.), polyclonal rabbit JNK (1:1,000; Cell Signaling Technology, Inc.), polyclonal rabbit p-JNK (Thr183/Tyr185; 1:1,000; Cell Signaling Technology, Inc.), polyclonal rabbit ERK1/2 (137F5; 1:1,000; Cell Signaling Technology, Inc.), polyclonal rabbit p-ERK1/2 (Thr202/Tyr204; 1:1,000; Cell Signaling Technology, Inc.), polyclonal rabbit P38 (D13E1; 1:1,000; Cell Signaling Technology, Inc.), polyclonal rabbit p-P38 (Thr180/ Tyr182; 1:1,000; Cell Signaling Technology, Inc.), polyclonal rabbit Ras (27H5; 1:1,000; Cell Signaling Technology, Inc.), and monoclonal mouse $\beta$-actin (T0022; 1:5,000; Cell Signaling Technology, Inc.) at $4^{\circ} \mathrm{C}$. Following incubation, the membrane was rinsed with Tris-buffered saline with Tween-20 (TBST) for $15 \mathrm{~min}$ three times, and incubated with secondary antibody. The membrane was agitated for $1 \mathrm{~h}$ at room temperature, washed again in TBST, and were developed using an ECL Plus Western Blotting Detection System (Bio-Rad Laboratories, Inc., Hercules, CA, USA).

Kinase inhibitors and MTT assay. PD98059, a special inhibitor of the ERK1/2, was from (Sigma Company), 

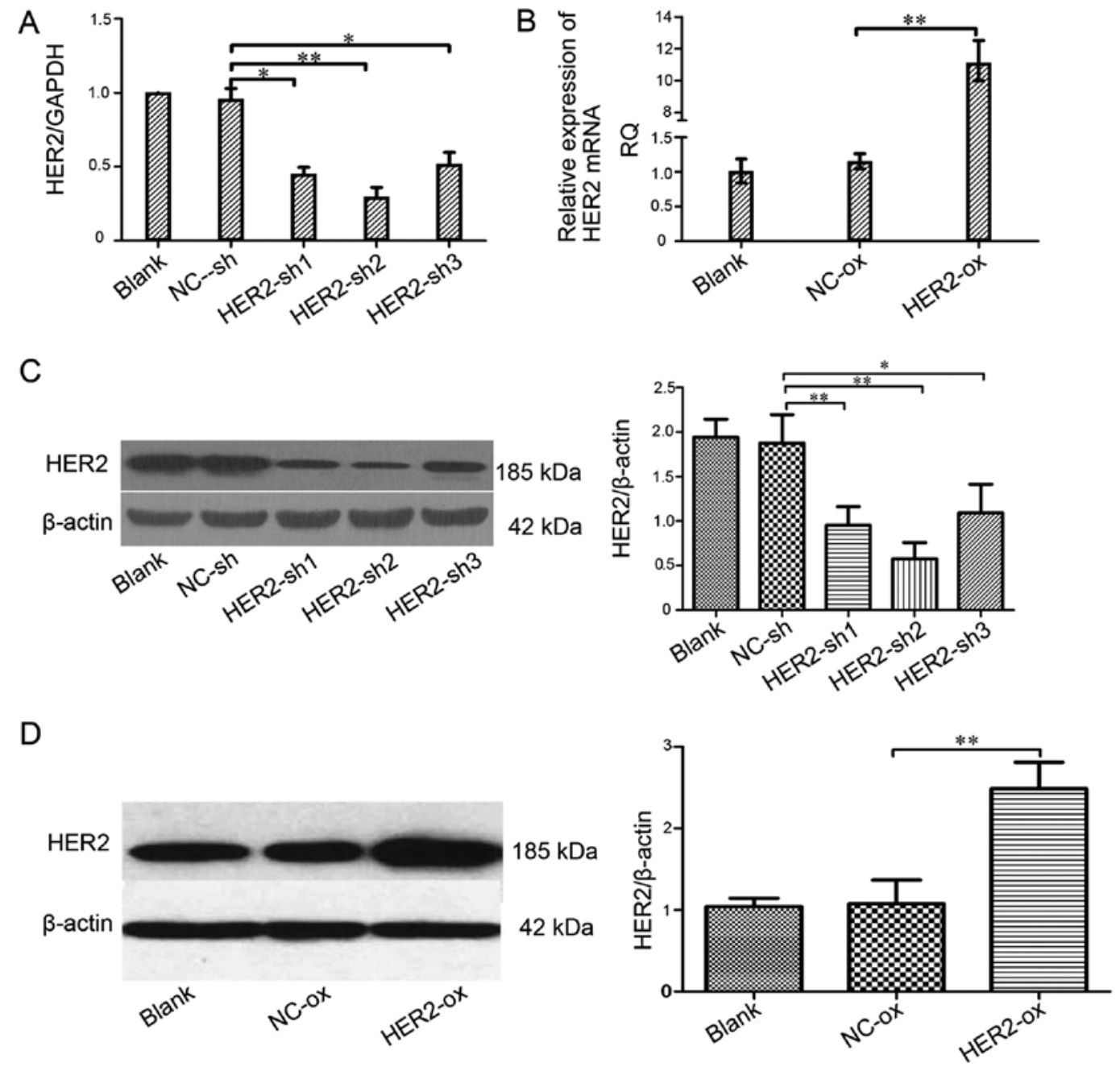

Figure 1. Effect of the HER-2 gene on the expression of HER-2 in IOMM-Lee cells. (A) The levels of HER-2 in different cell lines. Compared with NC-sh, the levels of HER-2 in the IOMM-Lee cell lines transfected with HER2-sh1, HER2-sh2, HER2-sh3 were decreased, obviously HER2-sh2 ("*P<0.01). (B) The mRNA levels of HER-2 in the IOMM-Lee malignant meningioma cells. Compared with the NC-overexpression, the expression of HER-2 in the IOMM-Lee cells transfected with HER-2-over was significantly increased (** $\mathrm{P}<0.01)$. (C and D) The protein expression of HER-2 in IOMM-Lee cells transfected with HER-2-sh and HER-2-over were analyzed by western blotting. (C) The protein expression levels of HER-2 in the IOMM-Lee cell lines transfected with HER-2-sh 2 obviously decreased ( $\left.{ }^{* *} \mathrm{P}<0.01\right)$. The data are expressed as the mean standard deviation from three independent experiments. (D) The protein expression levels of HER-2 in the IOMM-Lee cell lines transfected with HER-2-over obviously increased $\left({ }^{* *} \mathrm{P}<0.01\right)$. The data are expressed as the mean standard deviation from three independent experiments. Blank, blank control; NC, negative; sh, short hairpin, over, overexpression.

SP600125, the inhibitor of JNK, was from (Sigma Company), XMD8-92, an effective inhibitor of the ERK5, from (Santa Cruz Biotechnology). Treated with different concentration of ERK1/2 inhibitor, JNK inhibitor, ERK5 inhibitor, cell proliferation ability of each group were assessed using MTT assay. Briefly, the exponential growth phase, were trypinized, centrifuged at $400 \mathrm{x} \mathrm{g}$ for $5 \mathrm{~min}$ at room temperature, resuspend in complete medium (10\% FBS) and were counted. The cells were then seeded into five 96 -well plates $\left(1 \times 10^{3}\right.$ cells/well), with five parallel wells for each cell group. The medium was replaced with $120 \mu 1$ MTT solution, containing $100 \mu 1$ medium and $20 \mu \mathrm{l}$ MTT (Beijing Solarbio Science \& Technology Co., Ltd.), at different time-points $(24,48,72 \mathrm{~h})$. After $4 \mathrm{~h}$, the medium was aspirated and $150 \mu \mathrm{l}$ DMSO was added to each well, then the plate was agitated for $45 \mathrm{sec}$ at $27^{\circ} \mathrm{C}$. The optical density value at a wavelength of $490 \mathrm{~nm}$ was determined using a Multiskan FC Microplate photometer (Thermo Fisher Scientific, Waltham, MA, USA).
Cell invasion analysis. The invasion assays were performed using Transwell inserts (Merck Millipore, Billerica, MA, USA; $8 \mu \mathrm{m}$ pore size) in 24 -well plates. Approximately $1 \times 10^{5}$ cells in $200 \mu \mathrm{l}$ of serum-free DMEM-F12 medium were placed in the upper chamber, and $500 \mu \mathrm{l}$ of the medium containing $15 \%$ FBS were placed in the lower chamber. For the invasion assay, Transwell membranes were pre-coated with Matrigel (BD Biosciences, Bedford, MA, USA). The cells were incubated for $36 \mathrm{~h}$ at $37^{\circ} \mathrm{C}$ in $5 \% \mathrm{CO}_{2}$. The cells were incubated in the uncoated Transwells for $24 \mathrm{~h}$. Then, the cells were fixed in $10 \%$ paraformaldehyde for $15 \mathrm{~min}$ and stained with $0.05 \%$ crystal violet in PBS for $30 \mathrm{~min}$. The cells on the upper side of the filters were removed with cotton-tipped swabs, and the filters were washed with PBS. The cells invaded through the filter were dried for $20 \mathrm{~min}$, fixed in absolute alcohol and stained with $8 \mathrm{~g} / 1$ hematoxylin and eosin. The cells on the underside of the filters were examined and counted with a total x100 magnification under a Leica DMI 4000 microscope. 

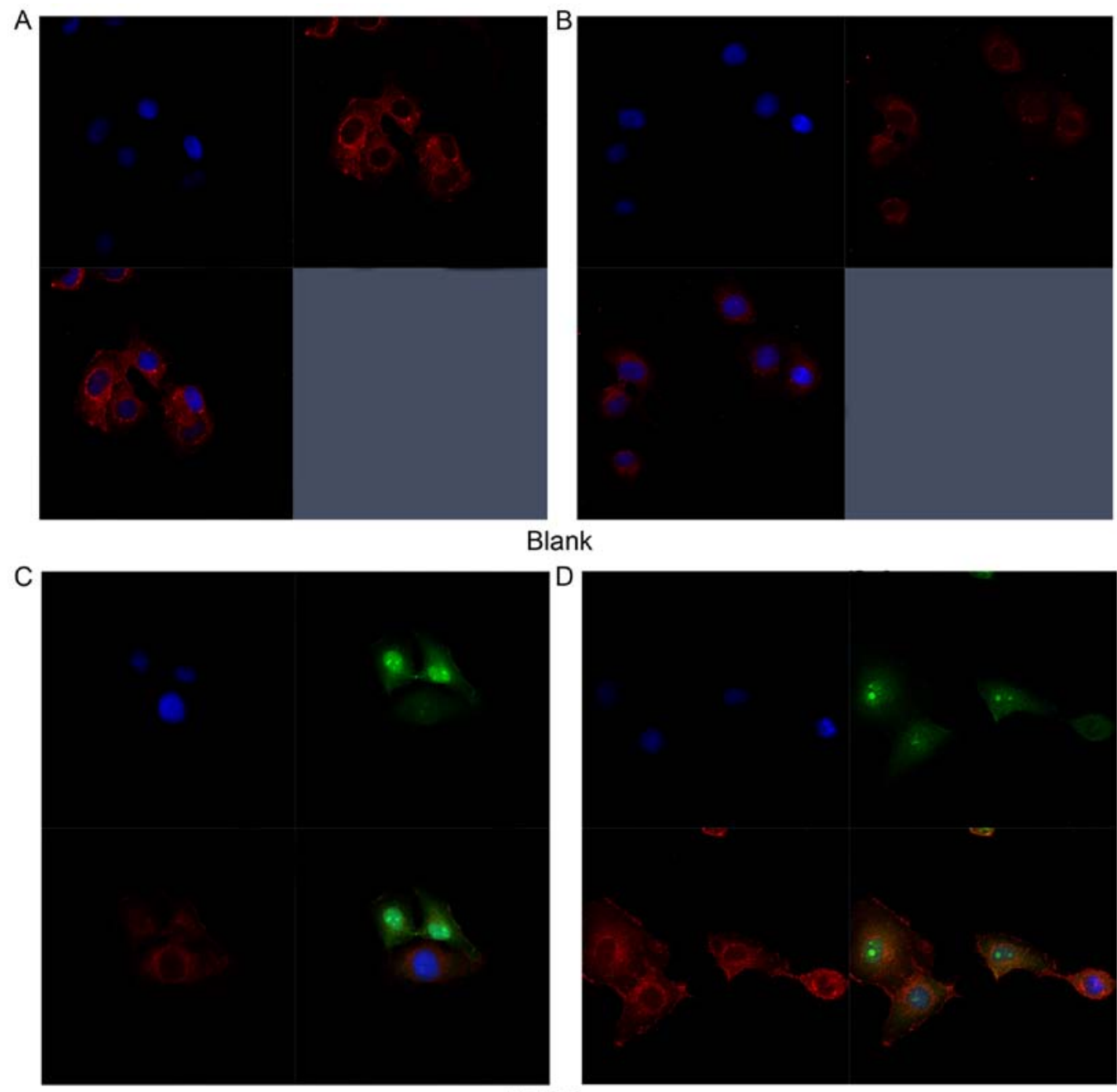

HER-2-over

Figure 2. The IOMM-Lee cells transfected with the HER-2-overexpression lentiviral reflected by immunofluorescence staining. (A and B) Immunofluorescence staining of HER-2 in the blank group showed HER-2 expression in the IOMM-Lee cells. (C and D) Immunofluorescence staining of HER-2 in the HER-2-over group presented strong effects on the IOMM-Lee cells transfected with HER-2-overexpression lentiviral. Blank, blank control; over, overexpression.

Each experiment was performed in triplicate and repeated at least three times.

Effects of ERK1/2, ERK5, JNK inhibitors on the proliferation and invasion of IOMM-Lee cells transfected with HER-2 overexpression lentiviral vector. The IOMM-Lee cells were treated with $10 \mu \mathrm{g} / 1,20 \mu \mathrm{g} / 1,40 \mu \mathrm{g} / 1$ PD98059 (ERK1/2 inhibitor), $10 \mu \mathrm{g} / 1,20 \mu \mathrm{g} / 1,40 \mu \mathrm{g} / 1 \mathrm{SP} 600125$ (JNK inhibitor), $5 \mu \mathrm{g} / 1$, $10 \mu \mathrm{g} / 1,20 \mu \mathrm{g} / 1 \mathrm{XMD} 8-92$ (ERK5 inhibitor) for $30 \mathrm{~min}$, respectively, and then with HER-2-overexpression cells or HER-2-NC cells for indicated periods. The ERK1/2, JNK and ERK5 expression was detected by western blot assay $30 \mathrm{~min}$ later, the cell invasion was detected $24 \mathrm{~h}$ later, and the cell proliferation was measured by MTT assay, then observed after $24,48,72 \mathrm{~h}$, respectively.

Animal models. A total of 30 female infant (4-6 weeks) $\mathrm{BALB} / \mathrm{c}$ nude mice, of specific pathogen-free grade, weighing 18-20 g, were used in this study. Rats were obtained from the SJA Laboratory Animal Co., Ltd. (Hunan, China). All nude mice were housed in the Laboratory Animal Center of the First Affiliated Hospital of Nanchang University (Nanchang,
Jiangxi, China). All nude mice were maintained in an airconditioned room with a 12-hour light/dark cycle, standard diet and water were available ad libitum. Ethical approval for this study was obtained from the Ethics Committee of Nanchang University. First, the nude mice were randomly separated into five groups ( $n=6 /$ group) (blank, NC-sh, HER-2-sh, NC-ox, HER-2-ox), After five days, the IOMM-Lee malignant cells were seeded for each groups. IOMM-Lee cells, in the exponential growth phase, were trypinized, at $400 \mathrm{x} \mathrm{g}$ for $5 \mathrm{~min}$ at room temperature, resuspending in complete medium (10\% FBS) and were counted for $5 \times 10^{7} / \mathrm{ml}$. Then IOMM-Lee cells ( $1 \times 10^{7} /$ mice) were injected subcutaneously into the right armpit of six-week-old nude mice, five mice in each group, and the blank control was nude mice injected equivalent serum-free DMEM medium. Close observation after injection, recorded the nude mice in vivo. The tumors were measured once every 4 days for the longest diameter a and shortest path $b$, according to the formula $\mathrm{V}=0.52 \mathrm{ab} 2$ calculation into the tumor size, rendering time-tumor volume growth curve. All the mice were sacrificed at day 30, the tumors were removed and calculated, the average tumor weight to calculate the inhibitory rate. Tumor inhibitory 
A
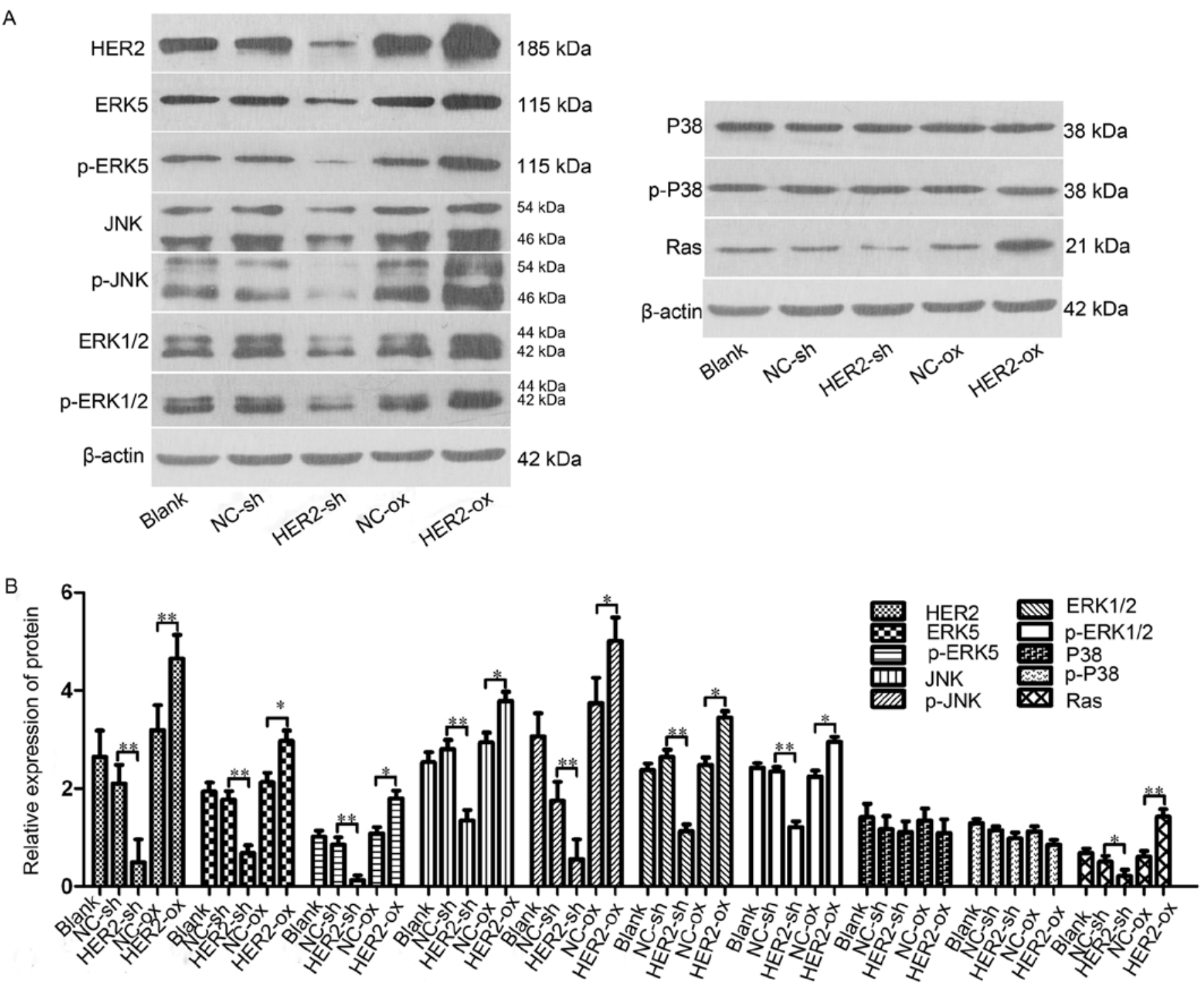

Figure 3. HER-2 affects protein expression levels of ERK5, JNK, ERK1/2, P38 and Ras in IOMM-Lee cells. (A) Protein levels of ERK5, p-ERK5, JNK, p-JNK, ERK1/2, p-ERK1/2, P38, p-P38, Ras in the IOMM-Lee cells were determined using western blot analysis $72 \mathrm{~h}$ post-transfection. $\beta$-actin was used as an internal loading control. (B) Protein levels of ERK5, p-ERK5, JNK, p-JNK, ERK1/2, p-ERK1/2, Ras in the HER-2-sh group were significantly decreased. However, in the HER-2-over group, the protein levels of ERK5, p-ERK5, JNK, p-JNK, ERK1/2, p-ERK1/2, Ras were increased, compared with NC control and following normalization against $\beta$-actin $(\mathrm{P}<0.05)$. No difference was observed between the protein expression of P38, p-P38 in the HER-2-sh and HER-2-over cells with that in the $\mathrm{NC}$ control. The data are expressed as the mean standard deviation from three independent experiments. NC, negative control; sh, short hairpin; over, overexpression; ERK5, extracellular signal-regulated kinase 5; JNK, Jun N-terminal kinase; ERK1/2, extracellular regulated protein kinases 1/2; p-, phosphorylated.

rate $=($ control group - experimental tumor $) /$ control tumor weight by $100 \%$.

Statistical analysis. Data were expressed as mean \pm standard error of the mean (SEM), either Student's t-test or one-way analysis of variance (ANOVA) was used for statistical analysis. All analyses were performed using the Statistical Package for Social Sciences (SPSS) 17.0 software (SPSS, Chicago, IL, USA) and $\mathrm{P}<0.05$ was considered to be statistically significant.

\section{Results}

Expression of HER-2 in human malignant meningioma cell lines. To analyze the role of HER-2 in the IOMM-Lee cell lines, the present study assessed the expression of HER-2 in the IOMM-Lee cell lines applying q-PCR following transfection for $96 \mathrm{~h}$ (Fig. 1A and B). Compared with the mock or the cells transfected with NC-sh in the levels of HER-2, the HER-2-expression of transfected with HER-2-sh1, HER-2-sh2, HER-2-sh3 all in the IOMM-Lee cells decreased, but HER-2-sh 2 decreased $75.3 \%(\mathrm{P}<0.01)$. The HER-2expression in the HER-2-overexpression IOMM-Lee cells was significantly increased (8.7-fold), compared with the blank or $\mathrm{NC}$-overexpression cells $(\mathrm{P}<0.01)$. Next, we also found similar effects on the protein levels of HER-2, $72 \mathrm{~h}$ post-infection in the western blotting. As shown in Fig. $1 \mathrm{C}$, it revealed that the protein level of HER-2 in the HER-2-sh2 group significantly decreased, by $60.67 \%$. compared with the blank, NC cells or HER-2-sh1, HER-2-sh3 ( $\mathrm{P}<0.01)$. As shown in Fig. 1D the expression of HER-2 in the HER-2-overexpression 


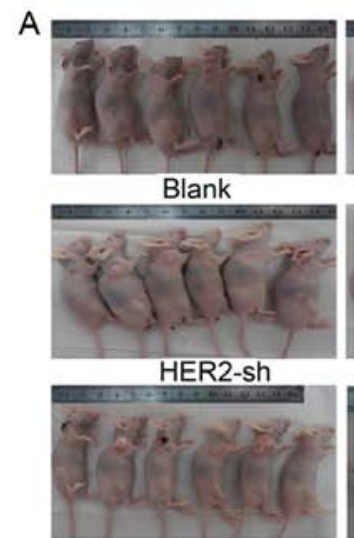

HER2-ox

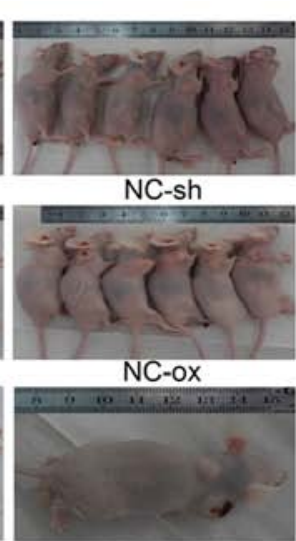

HER2-ox

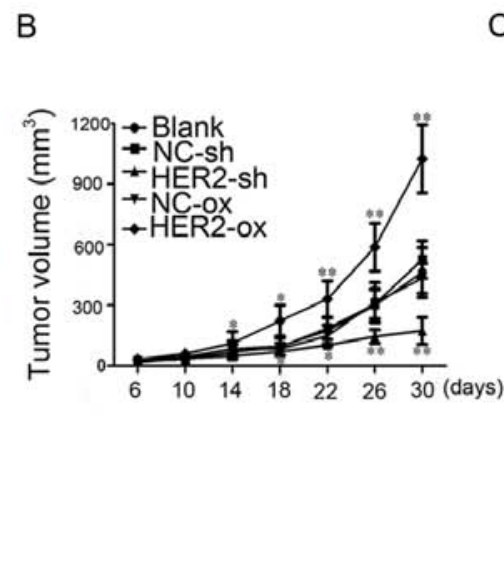

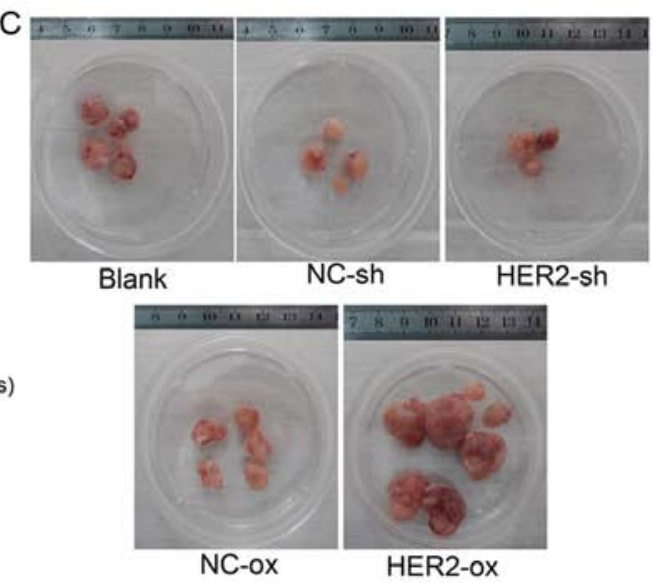

Figure 4. Tumorigenesis of HER-2-overexpression in IOMM-Lee cells in vivo. (A) Growth situation of tumor-burdened nude mice. (B) Time - a tumorburdened nude mouse tumor growth curve $\left({ }^{*} \mathrm{P}<0.05,{ }^{* *} \mathrm{P}<0.01\right)$. (C) Tumor number and volume of each group. Blank, blank control; NC, negative; sh, short hairpin, over, overexpression.

group increased (2.6-fold), compared with mock or NC cells $(\mathrm{P}<0.01)$. In the next experiments, we elected the HER-2-sh2 as the HER-2-sh group because of its high efficiency of transfection with the silence lentiviral vector.

IOMM-Lee cells transfected with the HER-2-overexpression lentiviral reflected by immunofluorescence. In Fig. 2A and B, the immunofluoresence results revealed the expression of HER-2 both cell surface and part of cytoplasm reflected by the red fluorescence in the blank group. As shown in Fig. 2C, the results suggested that HER-2 express in the cell surface in the IOMM-Lee cells transfected with HER-2-overexpression lentiviral vector refected by the green fluorescence, but the positive intensity of red fluoresence decreased, compared with the blank group. However, in Fig. 2D, the red fluorescence intensity significantly heighten suggested that the expression of HER-2 increased in the HER-2-overexpression IOMM-Lee cells, compared with blank group. Thus, we chose the HER-2overexpression cells for the further experiments.

HER-2 affects protein expression levels of ERK5, JNK, ERK1/2, P38 and Ras in IOMM-Lee cells without any inhibitors. In order to investigate the association between HER-2 and the activity of the Ras/MAPK signaling pahway, the present study measured the protein levels of ERK5, p-ERK5, JNK, p-JNK, ERK1/2, p-ERK1/2, P38, p-P38, Ras in the IOMM-Lee cells following transfection. The results shown that, compared with NC-sh group, the protein levels of ERK5, p-ERK5, JNK, p-JNK, ERK1/2, p-ERK1/2, Ras in the downregulated HER-2 group were all reduced, with significant difference between the two groups, with statistical significance $(\mathrm{P}<0.05$; Fig. $3 \mathrm{~A}$ and $\mathrm{B})$. By contrast, in the upregulated HER-2 group, the protein levels of ERK5, p-ERK5, JNK, p-JNK, ERK1/2, p-ERK1/2, Ras all increased $(\mathrm{P}<0.05$; Fig. 3A and B). However, no difference was observed in the protein expression of P38, p-P38 in these two groups, compared with NC group.

Tumorigenesis of HER-2-overexpression IOMM-Lee cells in vivo. Our results suggested that HER-2 can increase cell proliferation in vitro. To confirm this effect in vivo, the cells (blank, NC-sh, HER-2-sh, NC-ox, HER-2-ox) were subcutaneously inoculated into nude mice. The tumor was observed in the blank group, NC-sh group, HER-2-sh group, NC-ox group and HER-2-ox group on days four after subcutaneous inoculation showed there were 2, 3, 2, 2, 4 nude mice with solid mass, respectively, but liquidity bag piece of the blank group had disappeared. The tumor was observed in the blank group, NC-sh group, HER-2-sh group, NC-ox group and HER-2-ox group on days six after subcutaneously inoculation showed there were 4, 4, 3, 5, 5 nude mice with solid mass, respectively. On day 22, and 26 days after subcutaneous inoculation, in the HER-2-ox group one nude mouse had macroscopic transfer. All of the mice were sacrificed at days 30, the tumor at formation rate of $84 \%$ with on natural death (Fig. $4 \mathrm{~A}$ ). Close observation after injection, on days six, recorded the nude mice in vivo with the tumor, measured once every 4 days for the longest diameter and shortest path, calculated the tumor size, rendering time-tumor volume growth curve. Our result revealed tumor volume change at days 14 after subcutaneous inoculation. Volume growth of HER-2-sh decreased by $28.36 \%$ compared with NC-sh group, While the volume growth of HER-2-ox increased by $32.14 \%$ compared with the NC-ox group $(\mathrm{P}<0.05)$. After 14 days the volume significantly increased, the differences were statistically significant $(\mathrm{P}<0.01)$. Timetumor volume growth result revealed that HER-2 increased cell growth and proliferation in meningioma cells (Fig. 4B). Tumor volume was measured once every 4 days using a vernier caliper and the tumors were collected on day 30 . The mean volume of tumors in HER-2-sh group, NC-sh group, HER-2-ox group, NC-ox group was $139.33 \pm 13.89 \mathrm{mg}, 236.34 \pm 54.18 \mathrm{mg}$, $357.33 \pm 42.24 \mathrm{mg}$ and $223 \pm 36.16 \mathrm{mg}$, respectively. Tumor inhibitory rate of HER-2-sh group was $41.05 \%$ compared with NC-sh group, HER-2-ox group was $-55.64 \%$, the differences were statistically significant $(\mathrm{P}<0.01$; Fig. $4 \mathrm{C})$. The result also illustrated HER-2 increased the cell proliferation of malignant meningioma in vivo.

The role of MAPK pathway inhibitions PD98059 (ERK1/2 inhibitor), SP600125 (JNK inhibitor), XMD8-92 (ERK5 


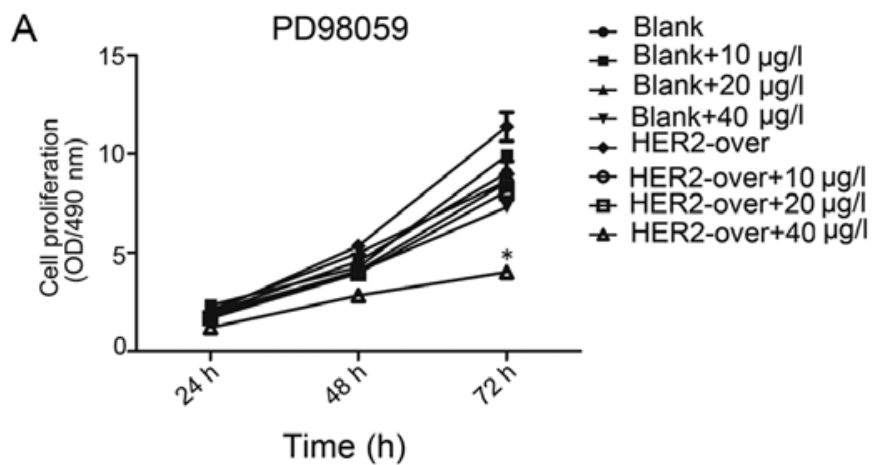

C

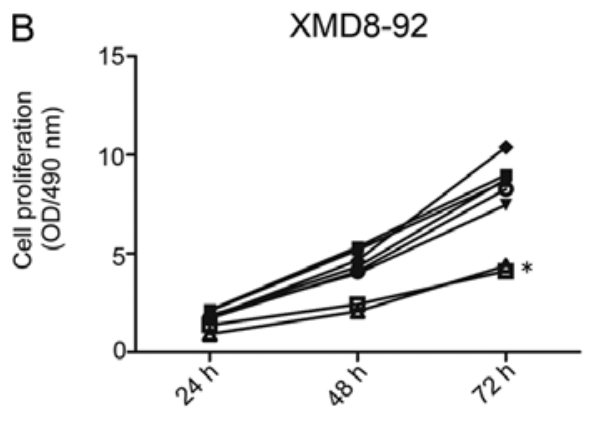

Time(h)

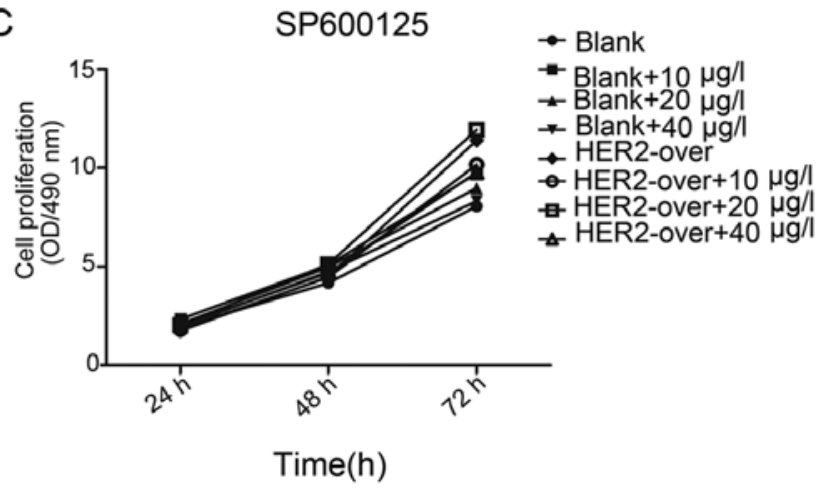

Figure 5. MTT assay present the inhibition of the cell proliferation. (A) MTT assay at $72 \mathrm{~h}$, in HER-2-over with concentration of $40 \mu \mathrm{g} / 1$ PD98059 (ERK1/2 inhibition) cell resistance was 1.54-fold higher than that of HER-2-over without inhibition $(\mathrm{P}<0.01)$, whereas the resistance of the HER-2-over with concentration of $40 \mu \mathrm{g} / 1$ of PD98059 (ERK1/2 inhibition) cells significantly decreased 45\%, compared with the blank group with the same concentration of PD98059 (P<0.01). (B) At $72 \mathrm{~h}$, HER-2-over with concentration of $10 \mu \mathrm{g} / 1$ XMD8-92 (ERK5 inhibition) cell resistance was decreased $73.8 \%$ compared with the HER-2-over without inhibition $(\mathrm{P}<0.01$ ), whereas the resistance of the HER-2-over with concentration of $10 \mu \mathrm{g} / 1$ XMD8-92 (ERK5 inhibition) cells significantly decreased 24\%, compared with the blank group with the same concentration PD98059 (P<0.01). (C) At different time-point (24, 48, $72 \mathrm{~h}$ ), MTT assay revealed that, no difference was observed in the groups with the different concentration of SP600125 (10, 20, $40 \mu \mathrm{g} / \mathrm{l})(\mathrm{P}>0.05)$. Blank, blank control; over, overexpression.

inhibitor) in inhibiting the proliferation and metastasis of IOMM-Lee cells through the MAPK pathway in vitro. Cell invasion plays a crucial role in the tumor metastasis. MTT assay result present that at $24,48,72 \mathrm{~h}$, the cell proliferation of the blank group and HER-2-ox group with the different concentration inhibition of PD98059, XMD8-92, SP600125, respectively. As shown in Fig. 5, The present study observed a significant decrease in proliferation following adding PD98059 in MTT assay. At $72 \mathrm{~h}$, with the concentration of 10 , $20,40 \mu \mathrm{g} / 1$ PD98059 cell resistance was observed. However, HER-2-over with concentration of $40 \mu \mathrm{g} / 1$ ERK1/2 inhibition (PD98059) cell resistance was 1.54 -fold higher than that of HER-2-over without inhibition $(\mathrm{P}<0.01)$. Whereas, the resistance of the HER-2-over with concentration of $40 \mu \mathrm{g} / 1$ of PD98059(ERK1/2 inhibition) cells decrease significantly $45 \%$, compared with the blank group with the same concentration PD98059 $(\mathrm{P}<0.01)$. Therefore, we elected $40 \mu \mathrm{g} / \mathrm{l}$ PD98059 as the best inhibition concentration for further experiments.

In the blank group and HER-2-over group with the different concentration of XMD8-92 (ERK5 inhibition), in each group was observed a certain degree of inhibition $(\mathrm{P}<0.01)$. However at $72 \mathrm{~h}, \mathrm{HER}-2$-over with concentration of $10 \mu \mathrm{g} / 1$ of XMD8-92 (ERK5 inhibition) cell resistance was significantly decreased $73.8 \%$, compared with the HER-2-over without inhibition $(\mathrm{P}<0.01)$. Whereas, the resistance of the HER-2-over with concentration of $10 \mu \mathrm{g} / 1$ XMD8-92 (ERK5 inhibition) cells significantly decreased $24 \%$, compared with the blank group with the same concentration PD98059 $(\mathrm{P}<0.01)$. Thus, in the next experiments we elected the concentration of $10 \mu \mathrm{g} / \mathrm{l}$ of XMD8-92 as the ideal inhibition concentration. In addition, we next used the SP600125 (JNK inhibition) in a similar way to investigate the cell proliferation. At different time-point (24, $48,72 \mathrm{~h}$ ), MTT assay revealed that, no difference was observed in the groups with the different concentration of XMD8-92 $(10,20,40 \mu \mathrm{g} / \mathrm{l})(\mathrm{P}>0.05)$.

Transwell invasion assays were employed to detect the invasion abilities mediated by PD98059, SP600125, XMD8-92 inhibitions, respectively. Results as shown in Fig. 6A demonstrate the invasion ability of PD98059 (ERK1/2 inhibition) group used with concentration of $40 \mu \mathrm{g} / 1$ significantly decreased 58.5\%, compared with the HER-2-over group $(\mathrm{P}<0.05)$. In addition, XMD8-92 (ERK5 inhibitor) group with concentration of $10 \mu \mathrm{g} / \mathrm{l}$ decreased $46.1 \%$ when compared with the HER-2-over group (Fig. 6B; P<0.05). However, no difference was observed in the invasion ability of SP600125 group contrast to the HER-2-over group (Fig. 6C).

MAPK inhibitors (PD98059, XMD8-92, SP600125) inhibited protein expression of ERK1/2 and ERK5 in HER-2overexpression IOMM-Lee meningioma cells. In order to investigate the effect of PD98059, XMD8-92, SP600125 on the protein expression of MAPK (ERK1/2) signaling pathway. The result revealed that, PD98059 (ERK1/2 inhibi- 


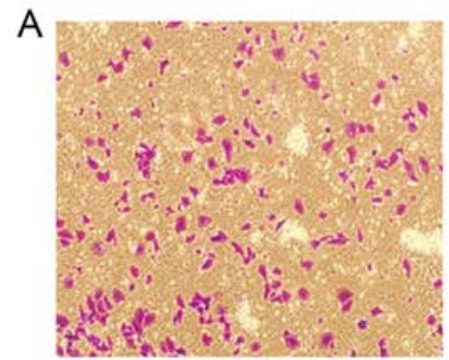

Blank

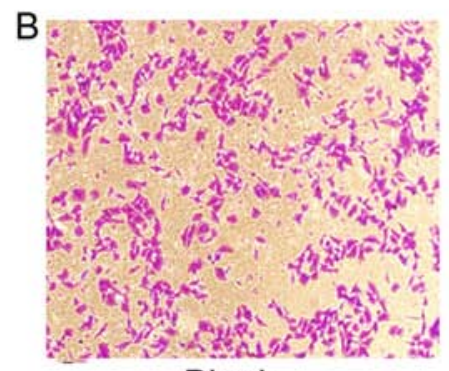

Blank

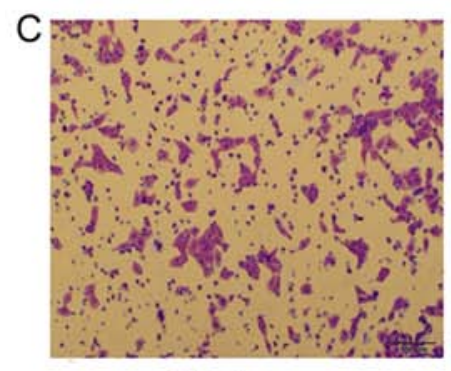

Blank

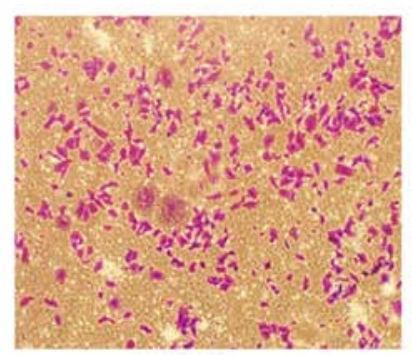

HER2-over

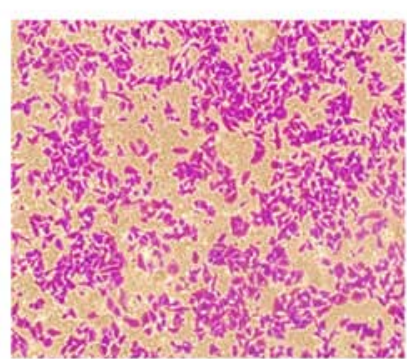

HER2-over

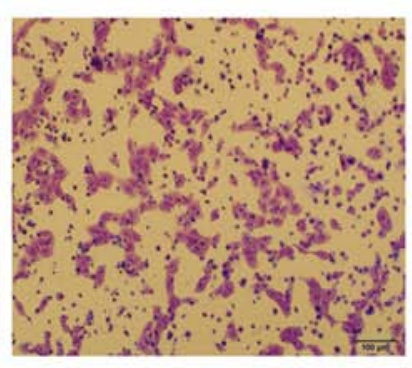

HER2-over

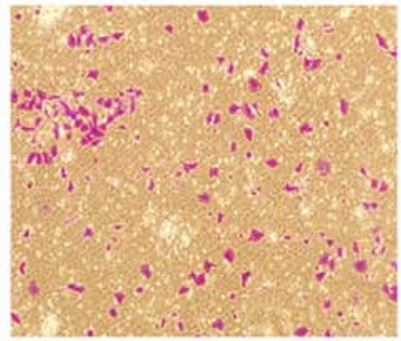

HER2-over+PD98059
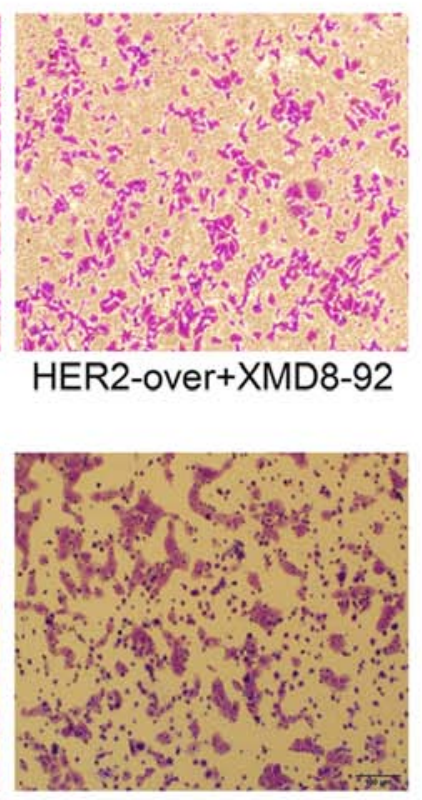

HER2-over+SP600125
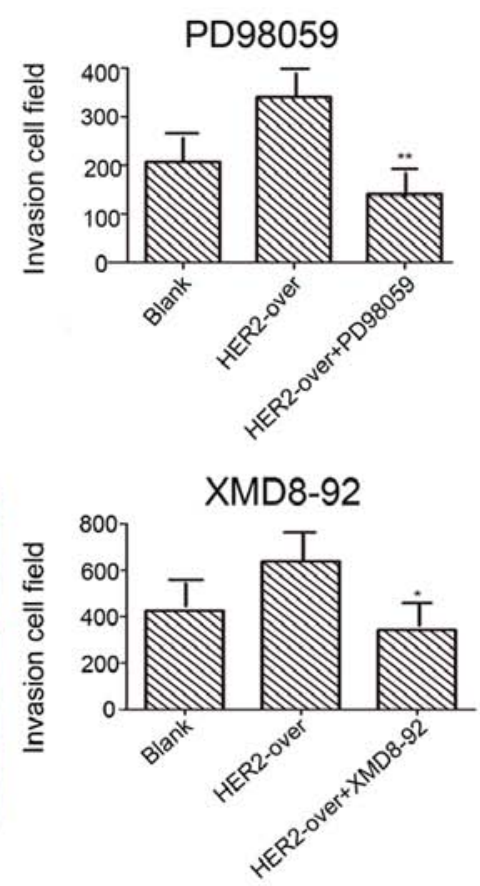

SP600125

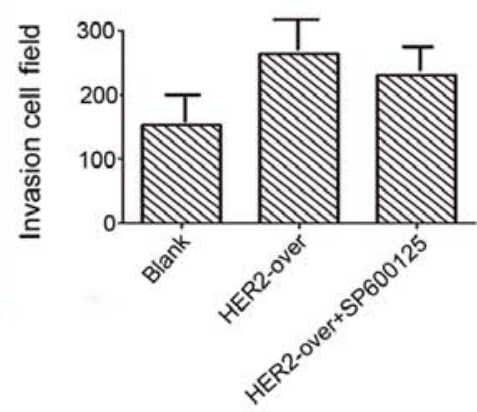

Figure 6. Inhibition of the IOMM-Lee cells transfected with the HER-over lentiviral vector cell invasion. (A) Representative images of Transwell invasion assays, demonstrating that invasion of HER-2-over cells with the concentration of $40 \mu \mathrm{g} / 1 \mathrm{PD} 98059$ was less than the HER-2-over group by 58.5\% (** $<0.05)$. (B) Transwell invasion assays with the concentration of $10 \mu \mathrm{g} / 1$ of XMD8-92 (ERK5 inhibition), the invasion of HER-2-over cell decreased 46.1\% ( $\mathrm{P}<0.05$ ). (C) No difference was observed in the invasion ability of SP600125 group contrast to the HER-2-over group (P>0.05). Blank, blank over, overexpression.

tion) inhibited the protein expression of ERK1/2 at the best inhibition concentration of $40 \mu \mathrm{g} / \mathrm{l}$ (Fig. 7A; $<<0.05)$. Western blot assay demonstrated XMD8-92 (ERK5 inhibition) significantly inhibited the protein expression of ERK5 the component of ERK1/2 signaling at the best inhibition concentration of $10 \mu \mathrm{g} / 1$ (Fig. 7B; $\mathrm{P}<0.01$ ). However, no effect was observed on the JNK at different inhibition concentration of SP600125 (Fig. 7C).

\section{Discussion}

HER-2 is a type of oncogene in human carcinoma, and many studies have indicated HER-2 overexpression in several types of cancer and is associated with a particularly aggressive form of the disease $(15,16)$. Our previous study demonstrated that overexpression of HER-2 and its mediated PI3K/AKT signaling pathway in patients with meningioma simulate human meningioma cell proliferation and invasion, which may contribute to its poor prognosis and development $(13,17)$.

In the present study, we constructed stable transformants cell lines (HER-2-overexpression and HER-2-sh) to investigate the expression level of HER-2 in human malignant meningioma cells, then examined with fluorescence microscopy, reverse transcription-quantitative polymerase chain reaction (q-PCR), Immunofluorescence (IF) staining and western blot analysis. The results reveal that the HER-2 mRNA and protein levels of HER-2-sh group cell significantly decreased over $50 \%$, while the HER-2-over group increased over 2-fold higher than of the blank group. In our previous study we demonstrated when the gene expression of HER-2 was downregulated, the proliferative ability of cells declined in vitro, determined using MTT assay (13).

Thus, in the present study we further investigated the proliferative ability of cells in vivo, determined using animal models. The result was consistent with the experiment in vitro that HER-2 gene downregulation declined the proliferative ability of cells. Next we analyzed the effect of PD98059, XMD8-92, SP600125 on the proliferation, metastasis and MAKP(ERK) signal pathway relevant protein expression in HER-2-overexpression in human malignant meningioma cells, determined using MTT assay, Transwell invasion assay and western blotting. Results showed that increased PD98059 
A

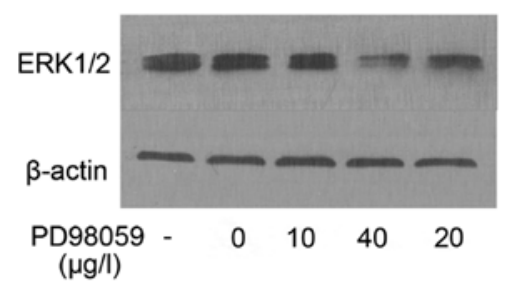

B

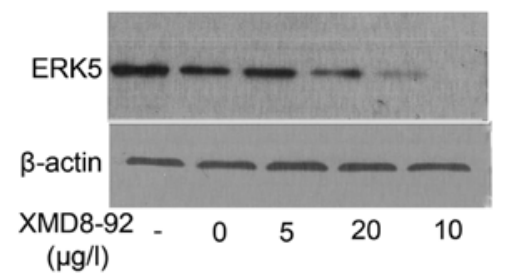

C

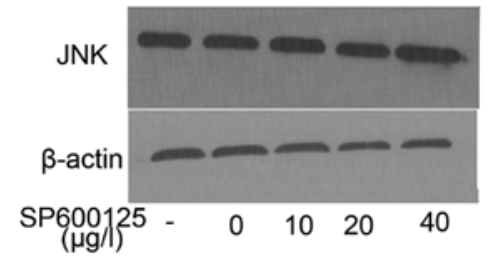

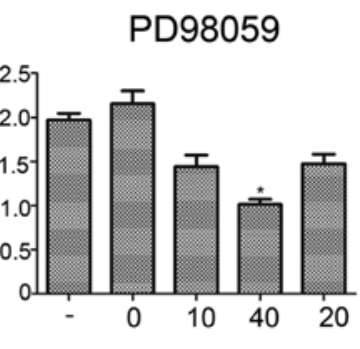
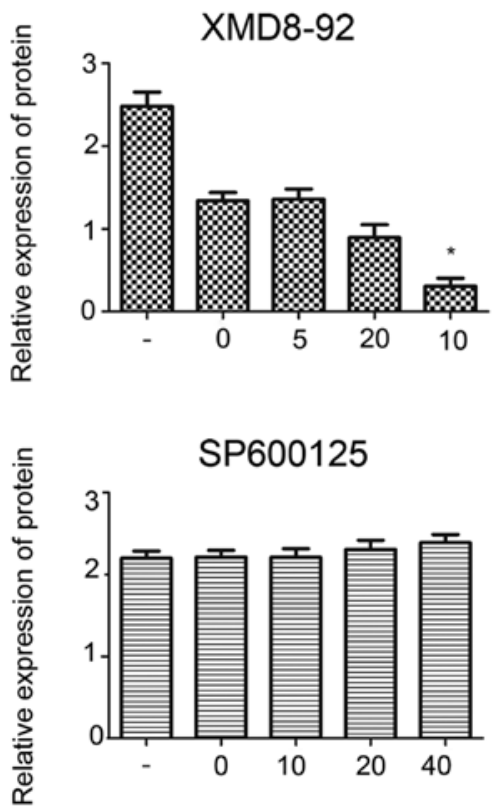

Figure 7. Inhibitions of protein expression of ERK1/2 and ERK5 through ERK1/2 (MAPK) pathways in HER-2-positive IOMM-Lee meningioma cells. Protein level of ERK1/2 in the HER-2-over IOMM-Lee cells were determined using western blot analysis at $72 \mathrm{~h}$ post-transfection. $\beta$-actin was used as an internal loading control. (A) Western blot assay demonstrated that PD98059 (ERK1/2 inhibition) inhibited the protein expression of ERK1/2 at the best inhibition concentration of $40 \mu \mathrm{g} / 1\left({ }^{*} \mathrm{P}<0.05\right)$. (B) Western blot assay demonstrated XMD8-92 (ERK5 inhibition) inhibited the protein expression of ERK5 the component of ERK1/2 signaling at the best inhibition concentration of $10 \mu \mathrm{g} / 1$ ( $\left.{ }^{*} \mathrm{P}<0.01\right)$. (C) No effect we can observed on the JNK at different inhibition concentration of SP600125. The data are expressed as the mean standard deviation from three independent experiments. -, Blank group; 0 , the inhibition of $0 \mu \mathrm{g} / 1 ; 5$, the inhibition of $5 \mu \mathrm{g} / 1 ; 10$, the inhibition of $10 \mu \mathrm{g} / 1 ; 20$, the inhibition of $20 \mu \mathrm{g} / 1 ; 40$, the inhibition of $40 \mu \mathrm{g} / 1$.

inhibition concentration inhibited the cell proliferation and invasion of HER-2-overexpression meningioma cells, the effect of XMD8-92 on the inhibition of cell proliferation ability of HER-2-overexpression meningioma cells compared with PD98059 was more potent and the inhibition effect of cell invasion was observed. However, no effect was observed in the cell proliferation and invasion of HER-2-overexpression meningioma cells of SP600125. In terms of western blotting, our results showed that PD98059 and XMD8-92 decreased the protein expression of ERK1/2 and ERK5, whereas SP600125 had no effect on the JNK. Therefore, the present study demonstrated that HER-2 promoted cell proliferation and invasion in the human malignant meningioma IOMM-Lee cells and provided some evidences for a functional linkage between HER-2 signaling and the activity of MAPK (ERK) in cell proliferation and invasion.

According to a previous study, HER-2 plays a role by homo- or heterodimerization with an extracellular domain (ECD) of other ErbB family members, which close proximity of the receptors leads to phosphorylation of the C-terminal tyrosines. Some phosphorylation sites are the tyrosines residues on the receptor molecule serving as recognition and docking sites for SH2-containing protein which consist of the components to activate the RAS/MAPK pathway and PI3K/ AKT pathway $(4,18,19)$.

The generic MAPK signaling pathway is shared by at least four distinct cascades, which are named according to their MAPK tier component: the extracellular signal-related kinase (ERK1/2), Jun amino-terminal kinases (JNK1/2/3), p38-MAPK and ERK5. MAPK pathway is an essential pathway in the cell proliferation, differentiation, migration, senescence and apoptosis (20). Therefore, based on the present study we speculate that HER-2 can affect the protein synthesis or activities of MAPK pathway, promote the cell proliferation and invasion. To assess this hypothesis, the present study used western blot analysis to determine the expression levels of MAPK pathway. Upregulation of the expression of HER-2 lead to increased levels of ERK1/2, ERK5 and JNK. ERK1/2 is pivotal in further signaling of the pathway, as it is reported that the GRB2 interacts with the guanine nucleotide exchange factor, SOS. SOS can then cause the exchange of guanosine diphosphate (GDP) to guanosine triphosphate (GTP) on RAS.

Activated RAS then initiates the activation of a kinase cascade culminating in the phosphorylation and activation 
of extracellular signal-regulated kinases 1 and 2 (ERK1, ERK2), where the ERK phosphorylates and activates various transcription factors, regulating various cellular processes, including proliferation, migration and differentiation $(21,22)$. Thus, the addition of PD98059 (ERK1/2) in the HER-2-overexpression meningioma cells suppressed cell proliferation and invasion. Next, ERK 5 is the effector kinase of a canonical three-tiered MAPK signalling cascade comprising MEK (MAPK/ERK kinase) 5, MEKK (MEK kinase) $2 / 3$ and ERK5 itself. The 444-amino-acid ERK5 protein, contains an $\mathrm{N}$-terminal kinase domain with $40 \%$ homology to the ERK2, and a large C-terminal extension that contains a transactivation domain.

ERK5 is activated via the dual phosphorylation of its Serine 311 and Threonine 315 by MEKK2, 3/Tp12, phosphorylates substrates including MEF2, c-Fos, Fra 1, Sap-1, $\mathrm{c}-\mathrm{Myc}$ and NF- $\mathrm{kB}$ that regulate the cell proliferation, survival, motility and angiogenesis. ERK5 and ERK1/2 present the cooperative effect in regulating some kinases (23-25). In other words, ERK5 and ERK1/2 signaling pathway may cross-talk on the regulating tumor cell proliferation and motility. As the present study results showed, inhibition of ERK5 also contributes to the impact on the cell proliferation and invasion abilities. Another component of the MAPK, the c-Jun NH2-terminal kinase (JNKs) is encoded by 2 ubiquitously expressed genes (Jnk1 and Jnk2) and by a third gene (Jnk3) can phosphorylate the c-Jun transcription factor at serine (Ser) 63 and -73 , resulting in the robust induction of c-Jun trans-activation. Previous studies demonstrated JNK is not required for proliferation and cell motility of mammary epithelial cells, but contributed to cell apoptosis. In addition, in individual tumor types, JNK may have an affect on tumor development or may contribute (positively or negatively) to tumor pathology. Further, owing to the poor selectivity of SP600125 for JNK, it is therefore unclear whether JNK inhibition mediates the effects of SP600125 on the proliferation and invasion of IOMM-Lee meningioma cells $(26,27)$. There are also other signal pathway, which infect the components of MAPK signal pathway (14). Thus, in the present study, ERK1/2, ERK5 and JNK may not be changed completely. We should provide insight into the exact molecular mechanisms in further study.

In summary, the present results suggested that overexpression of HER-2 promoted human meningioma cell proliferation and invasion in vivo and in vitro which may affect the meningioma development and progression. These results may explain, in part, the increased HER-2 in human meningioma cells which is clinically associated with the high recurrence potential and poor prognosis. Furthermore, the present study recorded the correlation between HER-2 signaling and the activity of MAPK (ERK) in cell proliferation and invasion. These data indicated that the HER-2-RASMAPK pathway to a certain extent may be available for further clinical development of human malignant meningioma for novel therapeutic approaches.

\section{Acknowledgements}

This study was supported by grants from the National Natural Science Foundation of China (grant no. 81260372) and the Science and Technology of Jiangxi Province (grant no. 20151BBG70217).

\section{References}

1. Louis DN, Ohgaki H, Wiestler OD, Cavenee WK, Burger PC, Jouvet A, Scheithauer BW and Kleihues P: WHO classification of tumours of the central nervous system. Acta Neuropathol 114: 97-109, 2007

2. Shibuya M: Pathology and molecular genetics of meningioma: Recent advances. Neurol Med Chir (Tokyo) 55: 14-27, 2015.

3. Jennifer M, Cope WP, Vartanian ED, Reiner AS, Kellen R, Ogilvie SQ, Huse JT and Gutin PH: Survival in patients treated for anaplastic meningioma. J Neurosurg 123: 23-30, 2015.

4. Cain SA, Smoll NR, Van Heerden J, Tsui A and Drummond KJ: Atypical and malignant meningiomas: Considerations for treatment and efficacy of radiotherapy. J Clin Neurosci 22: 1742-1748, 2015.

5. Dittrich A, Gautrey H, Browell D and Tyson-Capper A: The HER2 signaling network in breast cancer - Like a spider in its web. J Mammary Gland Biol Neoplasia 19: 253-270, 2014.

6. Tai W, Mahato R, Cheng K: The role of HER2 in cancer therapy and targeted drug delivery. J Control Release 146: 264-275, 2010.

7. Ménard S, Casalini P, Campiglio M, Pupa SM and Tagliabue E: Role of HER2/neu in tumor progression and therapy. Cell Mol Life Sci 61: 2965-2978, 2004.

8. Vernimmen D, Gueders M, Pisvin S, Delvenne P and Winkler R: Different mechanisms are implicated in ERBB2 gene overexpression in breast and in other cancers. Br J Cancer 89: 899-906, 2003.

9. Mahzouni P and Movahedipour M: An immunohistochemical study of HER 2 expression in meningioma and its correlation with tumor grade. Pathol Res Pract 208: 221-224, 2012.

10. Schwechheimer K, Läufle RM, Schmahl W, Knödlseder M, Fischer $\mathrm{H}$ and Höfler $\mathrm{H}$ : Expression of neu/c-erbB-2 in human brain tumors. Hum Pathol 25: 772-780, 1994.

11. Schlegel J, Ullrich B, Stumm G, Gass P, Harwerth IM, Hynes NE and Kiessling M: Expression of the c-erbB-2-encoded oncoprotein and progesterone receptor in human meningiomas. Acta Neuropathol 86: 473-479, 1993.

12. Chozick BS, Benzil DL, Stopa EG, Pezzullo JC, Knuckey NW, Epstein MH,Finkelstein SD and Finch PW: Immunohistochemical evaluation of erbB-2 and p53 protein expression in benign and atypical human meningiomas. J Neurooncol 27: 117-126, 1996.

13. Wang W, Tu Y, Wang S, Xu S, Xu L, Xiong Y, Mei J and Wang C: Role of HER-2 activity in the regulation of malignant meningioma cell proliferation and motility. Mol Med Rep 12: 3575-3582, 2015.

14. De Luca A, Maiello MR, D'Alessio A, Pergameno M and Normanno N: The RAS/RAF/MEK/ERK and the PI3K/AKT signalling pathways: role in cancer pathogenesis and implications for therapeutic approaches. Expert Opin Ther Targets 16 (Suppl 2): S17-S27, 2012.

15. Richman SD, Southward K, Chambers P, Cross D, Barrett J, Hemmings G, Taylor M, Wood H, Hutchins G, Foster JM, et al: HER2 overexpression and amplification as a potential therapeutic target in colorectal cancer: Analysis of 3256 patients enrolled in the QUASAR, FOCUS and PICCOLO colorectal cancer trials. J Pathol 238: 562-570, 2016.

16. Ren W, Liu Y, Wan S, Fei C, Wang W, Chen Y, Zhang Z, Wang T, Wang J, Zhou L, et al: BMP9 inhibits proliferation and metastasis of HER2-positive SK-BR-3 breast cancer cells through ERK1/2 and PI3K/AKT pathways. PLoS One 9: e96816, 2014.

17. Wang CL, Mei JH, Wang SS, Xu S, Xu LL and Xiong YF: Expression of HER2/neu in meningiomas: An immunohistochemistry and fluorescence in situ hybridization study. Zhonghua Bing Li Xue Za Zhi 39: 156-160, 2010 (In Chinese).

18. Yang L, Li Y and Zhang Y: Identification of prolidase as a high affinity ligand of the ErbB2 receptor and its regulation of ErbB2 signaling and cell growth. Cell Death Dis 5: e1211, 2014.

19. Elster N, Collins DM, Toomey S, Crown J, Eustace AJ and Hennessy BT: HER2-family signalling mechanisms, clinical implications and targeting in breast cancer. Breast Cancer Res Treat 149: 5-15, 2015.

20. Sun Y, Liu WZ, Liu T, Feng X, Yang N and Zhou HF: Signaling pathway of MAPK/ERK in cell proliferation, differentiation, migration, senescence and apoptosis. J Recept Signal Transduct Res 35: 600-604, 2015. 
21. Pearson G, Robinson F, Beers Gibson T, Xu BE, Karandikar M, Berman K and Cobb MH: Mitogen-activated protein (MAP) kinase pathways: Regulation and physiological functions. Endocr Rev 22: 153-183, 2001.

22. Rubinfeld $H$ and Seger R: The ERK cascade: A prototype of MAPK signaling. Mol Biotechnol 31: 151-174, 2005.

23. Lochhead PA, Gilley R and Cook SJ: ERK 5 and its role in tumour development. Biochem Soc Trans 40: 251-256, 2012.

24. Hu B, Ren D, Su D, Lin H, Xian Z, Wan X, Zhang J, Fu X, Jiang L, Diao D, et al: Expression of the phosphorylated MEK 5 protein is associated with TNM staging of colorectal cancer. BMC Cancer 12: 127, 2012.
25. Wang $X$ and Tournier C: Regulation of cellular functions by the ERK5 signalling pathway. Cell Signal 18: 753-760, 2006.

26. Cellurale C, Girnius N, Jiang F, Cavanagh-Kyros J, Lu S, Garlick DS, Mercurio AM and Davis RJ: Role of JNK in mammary gland development and breast cancer. Cancer Res 72: 472-481, 2012.

27. Li W, Wen C, Bai H, Wang X, Zhang X, Huang L, Yang X, Iwamoto A and Liu H: JNK signaling pathway is involved in piperlongumine-mediated apoptosis in human colorectal cancer HCT116 cells. Oncol Lett 10: 709-715, 2015. 